\title{
Perfil clínico-patológico de pacientes con pólipos vesiculares en la colecistectomía, en un hospital general de tercer nivel de Lima, Perú
}

\author{
Josué Aliaga Ramos, ${ }^{1}$ Carlos Barreda Costa, ${ }^{2}$ Martín Tagle Arrospide, ${ }^{3}$ José Pinto Valdivia, ${ }^{4}$ \\ Miluska Aliaga Ramos ${ }^{5}$
}

\footnotetext{
${ }^{1}$ Médico gastroenterologo-endoscopista, Servicio de Gastroenterologia Clinica San Pablo-Surco.

${ }^{2}$ Médico gastroenterologo-endoscopista, Servicio de Gastroenterologia Clinica Ricardo Palma.

${ }^{3}$ Médico gastroenterologo-hepatologo, Servicio de Gastroenterologia, Clinica Anglo-Americana.

${ }^{4}$ Médico gastroenterologo-endoscopista, Servicio de Gastroenterologia, Hospital Cayetano Heredia.

${ }_{5}^{5}$ Cirujana general-laparoscopista, Servicio de Cirugia General, Hospital Cayetano Heredia.

Lima, Perú.
}

Acta Gastroenterol Latinoam 2021;51(1):64-69

Recibido: 30/08/2020 / Aceptado: 16/02/2021 / Publicado online: 22/03/2021 / https://doi.org/10.52787/jxot9062

\begin{abstract}
Resumen
Introducción y objetivos. Las afecciones de la vesicula biliar son frecuentes en la práctica diaria. Algunas suelen ser subdiagnosticadas, como es el caso de los pólipos vesiculares, que muchas veces aparecen como hallazgo incidental al realizar una ultrasonografía abdominal o al examinar una vesicula extirpada por otra indicación. Sin embargo, pueden llegar a tener un impacto clínico muy importante si no se da un abordaje diagnóstico y terapéutico adecuado. El objetivo del presente estudio es describir el perfil clínico-patológico de los pacientes con pólipos vesiculares, poniendo énfasis en los parámetros considerados como factores de riesgo para transformación maligna de los pólipos neoplásicos. Material y métodos. Estudio descriptivo-retrospectivo. Se incluyeron los datos de pacientes que habian sido colecistectomizados en un
\end{abstract}

Correspondencia: Josué Aliaga Ramos

Correo electrónico: arjosue3000@gmail.com hospital general, en Lima-Perú entre junio de 2013 y junio de 2016, por diferentes patologías de la vesicula biliar. Se registraron la edad, el sexo, la sintomatología y los hallazgos de la ultrasonografía en los pacientes con pólipos vesiculares. Se calculó la tasa global de pólipos vesiculares y sus diferentes subtipos histológicos con sus respectivas características. Resultados. En el periodo descrito se incluyeron 1712 colecistectomías y en 162 (9,4\%) se encontraron pólipos vesiculares: colesterolósicos en 119 (86,9\%), hiperplásicos en 10 (7,3\%) $y$ adenomatosos en 8 (5,8\%). Se identificó que 108 (90,8\%) de los pólipos colesterolósicos fueron menores de $10 \mathrm{~mm} y$ $6(75 \%)$ de los adenomatosos fueron mayores de $10 \mathrm{~mm}$. Conclusión. En este estudio observamos que la mayoría de los pólipos vesiculares resultaron asintomáticos, benignos y de buen pronóstico. Casi un 6\% de los pólipos resultaron ser adenomatosos, subtipo que requiere una mayor atención por sus implicancias pronósticas.

Palabras claves. Pólipos vesiculares, pólipos neoplásicos, colecistectomía.

\section{Clinocopathological Profile of Patients with Gallbladder Polyps at Cholecystectomy in a Tertiary-Level Hospital in Lima, Peru}

\section{Summary}

Introduction and aims. Gallbladder conditions are common in daily practice. Some are usually underdiagnosed, as 
is the case of gallbladder polyps, which often appear as an incidental finding when performing an abdominal ultrasound or when examining a gallbladder removed for another indication. However, they can have an especially important clinical impact, if an adequate diagnostic and therapeutic approach is not given. The aim of the present study is to describe the clinical-pathological profile of patients with gallbladder polyps, emphasizing the parameters considered as risk factors for malignant transformation of neoplastic polyps. Materials and methods. Descriptive-retrospective study. The patients were cholecystectomized in a general hospital, in Lima-Peru between June of 2013 and June of 2016, for different pathologies of the gallbladder. Age, sex, symptoms, and the ultrasound findings of the patients with gallbladder polyps. Calculating the global rate of gallbladder polyps and their different histological subtypes with their respective characteristics. Results. In the described period, 1712 cholecystectomies were included and in 162 (9.4\%) gallbladder polyps were found: cholesterol in 119 (86.9\%), hyperplasic in 10 (7.3\%) and adenomatous in 8 (5.8\%). It was identified that 108 (90.8\%) of the cholesterol polyps were smaller than $10 \mathrm{~mm}$ and 6 (75\%) of the adenomatous polyps were larger than $10 \mathrm{~mm}$. Conclusion. In this study, we observed that most gallbladder polyps were asymptomatic, benign, and had a good prognosis. Almost 6\% of the polyps were found to be adenomatous, a subtype that requires further attention due to its prognostic implications.

Keywords. Gallbladder polyps, neoplastic polyps, cholecystectomy.

\section{Introducción y objetivos}

La prevalencia actual de los pólipos vesiculares, según estudios recientes, varía entre el 1\% al 5\% del total de colecistectomías por diversas patologías de la vesícula biliar. Sin embargo, diferentes grupos de investigación coinciden que esta entidad clínica está subdiagnosticada, debido principalmente al carácter asintomático en la mayoría de casos. Frecuentemente son un hallazgo incidental durante una colecistectomía por otra indicación médica o al realizar una ecografía abdominal de rutina. ${ }^{1-6}$ Los pólipos vesiculares se clasifican en dos grandes grupos: pólipos no neoplásicos y neoplásicos, los cuales representan el $87,1 \%$ y el $12,9 \%$ del total, respectivamente, siendo los pólipos de colesterol (no neoplásicos) y los adenomas (neoplásicos) los subtipos más representativos de cada uno de ellos. ${ }^{7-11}$

El impacto clínico de esta patología radica fundamentalmente en el potencial oncogénico de algunos pólipos adenomatosos, los cuales pueden degenerar a un ade- nocarcinoma de vesícula biliar en aproximadamente el 3-8\% de los casos. ${ }^{12-22}$ La ecografía abdominal es el examen auxiliar de primera línea para la detección de este tipo de lesiones, por su bajo costo, fácil disponibilidad y alta sensibilidad diagnóstica. Sin embargo, los nuevos avances en la endoscopía moderna han permitido que la ultrasonografía endoscópica optimice el diagnóstico y la caracterización de los pólipos neoplásicos, con la finalidad de ofrecer un mejor enfoque terapéutico. ${ }^{23-30} \mathrm{El}$ objetivo principal del presente estudio es describir el perfil clínicopatológico de los pacientes con pólipos vesiculares, así como también determinar la tasa de pólipos mayores de $10 \mathrm{~mm}$ y los demás factores de riesgo para transformación maligna de este tipo de lesiones.

\section{Material y métodos}

Estudio descriptivo-retrospectivo cuya información fue recolectada a partir de un banco de datos generado prospectivamente, realizado en el Hospital Cayetano Heredia, en el cual se incluyó a todos los pacientes con diagnóstico histopatológico confirmado de pólipo vesicular, los cuales habían sido sometidos a colecistectomía por diferentes patologías de la vesícula biliar, en un hospital general del tercer nivel de atención en Lima-Perú entre Junio del 2013 y Junio del 2016. Posteriormente, se tomaron los datos del informe de anatomía patológica (diagnóstico histológico, presencia de colelitiasis, colesterolosis y tamaño del pólipo). Además, se procedió a revisar las historias clínicas para extraer los datos de filiación (edad y sexo), los datos clínicos (sintomatología) y los datos ecográficos (visualización o no del pólipo, tamaño, presencia de colelitiasis, pólipo único o múltiple). Se excluyó a los pacientes con historias clínicas incompletas o con un diagnóstico histológico dudoso para pólipo vesicular. Luego se calculó la tasa global de pólipos vesiculares respecto al total de colecistectomías, así como también la tasa de cada subtipo y de pólipos mayores de $10 \mathrm{~mm}$. También se analizaron los factores de riesgo para transformación maligna de este tipo de lesiones (edad, tamaño $>10 \mathrm{~mm}$, presencia de síntomas, pólipo único o múltiple y coexistencia de colelitiasis).

\section{Análisis estadístico}

La tabulación de datos se realizó utilizando el programa Microsoft Excel para Windows 2010 y el análisis estadístico se llevó a cabo empleando el programa SPSS versión 24.0, considerando un nivel de significancia del $5 \%$. Asimismo, en el análisis de nuestros datos se calcularon las frecuencias, proporciones y promedios para cada una de las variables categóricas en estudio. 


\section{Consideraciones éticas}

El estudio fue aprobado por el Comité de Ética de la Institución en donde se llevó a cabo, el cual obra en poder del autor de correspondencia. El Comité de Ética de la institución aprobó nuestro estudio debido a que cumplió con todas las normas establecidas para la investigación científica, incluyendo la confidencialidad de datos de cada uno de los pacientes enrolados.

\section{Resultados}

Durante el período de estudio mencionado se realizaron 1712 colecistectomías, encontrándose pólipos vesiculares en 162 pacientes (9,4\%). Fueron excluidos 25 pacientes por las siguientes razones: 17 por presentar historias clínicas incompletas y 8 por tener un diagnóstico histológico dudoso de pólipo vesicular. Además, se encontraron 5 pacientes con cáncer avanzado de vesícula biliar; ninguno de ellos tuvo diagnóstico preoperatorio de pólipo vesicular. La Tabla 1 muestra las características clínicas de los pacientes enrolados en el estudio.

De los 137 pacientes analizados, 104 fueron mujeres $(75,9 \%)$ y 33 fueron hombres (24\%). Respecto del subtipo de pólipo vesicular, predominaron los pólipos colesterolósi$\cos (\mathrm{n}=119)$, seguidos de los pólipos hiperplásicos $(\mathrm{n}=10)$, y finalmente los pólipos adenomatosos $(\mathrm{n}=8)$. La Tabla 2 muestra la distribución porcentual de estas lesiones.

De los 119 pacientes con pólipos colesterolósicos, la edad promedio fue de 42 años (rango: 15-70 años), 48,7\%
Tabla 2. Distribución porcentual de los subtipos de pólipos vesiculares

\begin{tabular}{lcc}
\hline & Nro. Casos & $\%$ \\
\hline Subtipo de pólipo vesicular & 119 & \\
Pólipos colesterolósicos & 10 & 86,90 \\
Pólipos hiperplásicos & 8 & 7,30 \\
Pólipos adenomatosos & 137 & 5,80 \\
Total & & 100 \\
\hline
\end{tabular}

(58 pacientes) eran asintomáticos, 76,5\% (91 pacientes) fueron pólipos únicos y el 90,8\% (108 pacientes) tuvieron un tamaño menor de $10 \mathrm{~mm}$. En cuanto a los pólipos adenomatosos, la edad promedio fue de 38,9 años (rango: 16-56 años), el 75\% (6 pacientes) eran sintomáticos, el $87,5 \%$ (7 pacientes) fueron pólipos únicos y un $75 \%$ (6 pacientes) eran mayores de $10 \mathrm{~mm}$.

Del total de los pólipos, solo el 12,4\% (17 pacientes) eran mayores de $10 \mathrm{~mm}$; de estos el 64,7\% (11 pacientes) eran pólipos colesterolósicos y el 35,2\% (6 pacientes) pólipos adenomatosos. La Tabla 3 muestra las características de las lesiones incluidas en el estudio.

\section{Discusión}

La principal fortaleza de nuestro estudio es que muestra, en una de las casuísticas más grandes de pólipos vesiculares en nuestro país, datos actualizados de una entidad

Tabla 1. Población y características clínicas

\begin{tabular}{lccc}
\hline Características de estudio & $\begin{array}{c}\text { Pólipos colesterolósicos } \\
(\mathrm{n}=119)\end{array}$ & $\begin{array}{c}\text { Pólipos hiperplásicos } \\
(\mathrm{n}=10)\end{array}$ & $\begin{array}{c}\text { Pólipos adenomatosos } \\
(\mathrm{n}=8)\end{array}$ \\
\hline Sexo & & & \\
Masculino & $27(22,7 \%)$ & $3(30 \%)$ & $3(37,5 \%)$ \\
Femenino & $92(77,3 \%)$ & $7(70 \%)$ & $5(62,5 \%)$ \\
Síntomas & & & \\
Asintomático & $58(48,7 \%)$ & $8(80 \%)$ & $2(25 \%)$ \\
Dolor en hipocondrio derecho & $59(49,6 \%)$ & $2(20 \%)$ & $6(75 \%)$ \\
Hiporexia & $5(4,2 \%)$ & $0(0 \%)$ & $0(0 \%)$ \\
Nauseas & $13(10,9 \%)$ & $0(0 \%)$ & $1(12,5 \%)$ \\
Hallazgos asociados & & & $6(75 \%)$ \\
Colesterolosis & $96(80,7 \%)$ & $9(90 \%)$ & $0(0 \%)$ \\
Colelitiasis & $1(0,8 \%)$ & $0(0 \%)$ & $6(75 \%)$ \\
Microlitiasis & $64(53,8 \%)$ & $2(20 \%)$ & $3(37,5 \%)$ \\
Otros & $29(24,4 \%)$ & $1(10 \%)$ & $38,9(16-56)$ \\
a Edad, años (IC 95\%) & $42,0(15-70)$ & $38,4(17-62)$ &
\end{tabular}


Tabla 3. Características de las lesiones

\begin{tabular}{lccc}
\hline Características de estudio $^{c}$ & $\begin{array}{c}\text { Pólipos colesterolósicos } \\
(\mathrm{n}=119)\end{array}$ & $\begin{array}{c}\text { Pólipos hiperplásicos } \\
(\mathrm{n}=10)\end{array}$ & $\begin{array}{c}\text { Pólipos adenomatosos } \\
(\mathrm{n}=8)\end{array}$ \\
\hline Numero de pólipos & $91(76,5 \%)$ & $10(100 \%)$ & $7(87,5 \%)$ \\
Pólipo único & $28(23,5 \%)$ & $0(0 \%)$ & $1(12,5 \%)$ \\
Pólipos múltiples & & & \\
Tamaño & $11(9,2 \%)$ & $0(0 \%)$ & $6(75 \%)$ \\
Mayor o igual a $10 \mathrm{~mm}$ & $108(90,8 \%)$ & $10(100 \%)$ & $2(25 \%)$ \\
Menor de 10 mm & &
\end{tabular}

clínica subdiagnosticada en la mayoría de los países latinoamericanos. Además, este estudio demuestra la importancia de no restringir la evaluación de estas lesiones únicamente al tamaño, mostrando otras condiciones de riesgo también asociadas a la transformación maligna de los pólipos vesiculares neoplásicos, poniendo énfasis en el análisis integral e individualizado de estos pacientes.

Los pólipos vesiculares son una entidad clínica diagnosticada frecuentemente como un hallazgo ecográfico y/o quirúrgico en pacientes asintomáticos que se realizan exámenes de rutina, dispépticos en estudio o aquellos sometidos a colecistectomía por alguna otra indicación quirúrgica. ${ }^{1-3}$ La incidencia actual de esta patología se está incrementando en los últimos años, según estudios elaborados recientemente; sin embargo, la magnitud de este aumento es altamente variable en distintas regiones. ${ }^{4-6}$

Diferentes grupos de investigación han encontrado diversas distribuciones porcentuales de los distintos subtipos de pólipos vesiculares, coincidiendo la mayoría en que los subtipos no neoplásicos son los más frecuentes. ${ }^{6-8}$ Uno de los estudios más grandes elaborados recientemente fue el publicado por $\mathrm{Xu}$ et al., ${ }^{9}$ quienes encontraron retrospectivamente en 1446 pacientes un 87,1\% (1260 pacientes) con pólipos de colesterol, 11,2\% (162 pacientes) fueron pólipos benignos no colesterolósicos y 1,7\% (24 pacientes) lesiones malignas, observando adicionalmente que más del $50 \%$ de los pólipos benignos no colesterolósicos (adenomas) presentaban un tamaño menor de $10 \mathrm{~mm}$. Asimismo evidenciaron que la edad mayor a 50 años, la presencia de síntomas, el tamaño mayor a $10 \mathrm{~mm}$ y la coexistencia de colelitiasis eran factores predictivos independientes para pólipos malignos. Uno de los estudios más recientes elaborados en Latinoamérica en en cual se logra replicar estos resultados fue el realizado por Bugosen et al., ${ }^{10}$ que observó en 1707 colecistectomías una prevalencia de pólipos vesiculares del 10\% (172 casos), de los cuales el 95,4\% (164 lesiones) fueron pólipos de colesterol, 4\% (7 lesiones) pólipos adenoma- tosos y 0,6\% (1 lesión) pólipos hiperplásicos; se registró únicamente el tamaño de 89 pólipos colesterolósicos, de los cuales el 69,7\% tenían un tamaño menor a $10 \mathrm{~mm}$. Asimismo se identificó el tamaño de solo 4 pólipos adenomatosos, de los cuales el $75 \%$ tenían un tamaño mayor o igual a $10 \mathrm{~mm}$. Nuestro estudio corrobora, al igual que los pocos trabajos de investigación elaborados en nuestro medio, los resultados de las dos investigaciones antes mencionadas.

La importancia de esta condición clínico-patológica es el potencial oncogénico a mediano-largo plazo de algunos pólipos vesiculares, lo que ha incentivado a múltiples investigadores a identificar los factores de riesgo asociados para esta transformación maligna. ${ }^{11-15}$ Onda et al. ${ }^{16}$ identificaron retrospectivamente como factores de riesgo para carcinoma en 141 pacientes con diagnóstico de pólipo vesicular al tamaño mayor o igual a $13 \mathrm{~mm}(p=0,032)$, la edad mayor a 65 años $(p=0,005)$, la morfología sésil $(p=0,124)$, el pólipo solitario $(p=0,300)$ y la coexistencia de colelitiasis $(p=0,075)$, presentando valores estadísticamente significativos en los dos primeros. Por el escaso número de pólipos adenomatosos y la ausencia de pólipos malignos en nuestra casuística, no se ha podido demostrar diferencias significativas, pero sí se ha observado una tendencia de los pólipos adenomatosos a ser únicos y de mayor tamaño, en relación con los de colesterol.

El enfoque terapéutico de los pólipos vesiculares es controversial; sin embargo, diferentes grupos de investigación han tratado de proponer distintos algoritmos con la finalidad de optimizar el tratamiento para este tipo de lesiones, teniendo en cuenta las características de riesgo establecidas por estudios previos. ${ }^{17-21}$ Recientemente, Bhatt et al. ${ }^{22}$ elaboraron un metaanálisis en el cual se incluyeron 54 estudios (6100 pacientes) en los que se estableció, mediante un análisis multivariado, que los pólipos vesiculares con un tamaño mayor o igual a $10 \mathrm{~mm}$ deberían ser resecados mediante una colecistectomía por presentar un alto riesgo de malignidad, y aquellos con un 
tamaño menor a $10 \mathrm{~mm}$ deberían ser sometidos a un seguimiento ecográfico (cada 2-3 años: tamaño $<4 \mathrm{~mm}$ y cada 6 meses: tamaño $4-10 \mathrm{~mm}$ ), observando las características de riesgo durante el seguimiento con la finalidad de ofrecer un tratamiento oportuno. Es importante resaltar que nuestra casuística muestra, al igual que otros estudios, algunos pacientes con pólipos colesterolósicos mayores de $10 \mathrm{~mm}$, lo que nos dice que la decisión de una conducta quirúrgica debe basarse en un enfoque individualizado de cada paciente.

En la actualidad, con la aparición de las nuevas técnicas endoscópicas, se ha logrado optimizar el diagnóstico de los pólipos vesiculares neoplásicos, lo cual es de gran importancia en pacientes de alto riesgo, ya que de esta forma se logra ofrecer una conducta terapéutica oportuna y adecuada. ${ }^{23,}{ }^{24}$ La ultrasonografía endoscópica es una de las técnicas diagnósticas que más ha sido sugerida por la mayoría de los autores como el mejor método para distinguir los pólipos vesiculares neoplásicos de los no neoplásicos. ${ }^{25-29} \mathrm{Kim}$ et al. ${ }^{30}$ identificaron prospectivamente en 202 pacientes con diagnóstico de pólipo vesicular, los cuales fueron sometidos a ultrasonografía endoscópica doppler-color, una exactitud diagnóstica para pólipos neoplásicos del $77,1 \%$, con una sensibilidad, especificidad, valor predictivo positivo y valor predictivo negativo del $89,2 \%, 41,6 \%, 27,4 \%$ y $94,1 \%$, respectivamente, encontrando además que el tamaño mayor o igual a $15 \mathrm{~mm}(p=0,012)$ y la presencia de un pólipo solitario $(p=0,002)$, identificados por este método diagnóstico, son factores predictivos para pólipos vesiculares neoplásicos con valores estadísticamente significativos. Esto nos demuestra que estas técnicas diagnósticas deberían ser utilizadas en pacientes con factores de riesgo para degeneración maligna de pólipos vesiculares, que a la vez presenten un alto riesgo quirúrgico, y de esta forma evitar cirugías innecesarias.

Nuestro estudio tiene algunas limitaciones; la primera es la población relativamente pequeña de pacientes enrolados, en comparación con los estudios más representativos. Sin embargo, en América Latina, así como en muchos países occidentales, la experiencia en validar el adecuado abordaje diagnostico-terapéutico de los pólipos vesiculares es aún limitada e inferior, en términos de uso de nuevas técnicas endoscópicas, a la reportada en países europeos y asiáticos. Otra limitación es que nuestros resultados representan la experiencia retrospectiva de un único centro, por lo que reconocemos que se necesitan estudios multicéntricos prospectivos a gran escala, con cohortes de pacientes más grandes y un tiempo de seguimiento más prolongado, para poder validar estos resultados a nivel mundial.

\section{Conclusión}

En conclusión, nuestro estudio muestra, al igual que investigaciones similares, que la mayoría de los pólipos vesiculares resultaron asintomáticos, benignos y de buen pronóstico. Casi un 6\% de los pólipos resultaron ser adenomatosos, subtipo que requiere una mayor atención por sus implicancias pronósticas. Futuros trabajos prospectivos, de mayor tamaño, de una población latinoamericana, permitirán tener datos más precisos sobre la epidemiología y el pronóstico de esta entidad.

Agradecimientos. Agradecemos al Dr. Carlos Barreda Costa del Servicio de Gastroenterologia de la Clinica Ricardo Palma por revisar tan amable y minuciosamente este manuscrito, asi como tambien al Jefe del servicio de Cirugia General del Hospital Cayetano Heredia por su buena disposicion para la elaboracion del presente estudio.

Conflictos de interés. Los autores declaran no tener ningún tipo de conflictos de interés.

Sostén financiero. Los autores declaran que para la elaboración del presente trabajo de investigación no se recibió ningún tipo de financiamiento por parte de ninguna institución pública o privada.

\section{Aviso de derechos de autor}

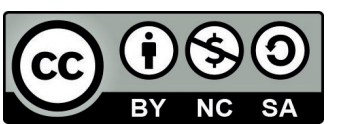

(C) 2021 Acta Gastroenterológica Latinoamericana. Este es un articulo de acceso abierto publicado bajo los términos de la Licencia Creative Commons Attribution (CC BY-NC-SA 4.0), la cual permite el uso, la distribución y la reproducción de forma no comercial, siempre que se cite al autor y la fuente original.

Cite este artículo como: Aliaga Ramos J, Barreda Costa C, Tagle Arrospide My col. Perfil clínico-patológico de pacientes con pólipos vesiculares en la colecistectomía, en un hospital general de tercer nivel de Lima, Perú. Acta Gastroenterol Latinoam. 2021;51(1):64-9. https://doi.org/10.52787/ jxot9062

\section{Referencias}

1. Pickering O, Pucher P, Toale C, et al. Prevalence and sonographic detection of gallbladder polyps in a Western European population. Journal of Surgical Research. 2020;250:226-31.

2. Dilek O, Karasu S, Dilek F. Diagnosis and treatment of gallbladder polyps: Current perspectives. Euroasian Journal of Hepato-Gastroenterology. 2019;9(1):40-8. 
3. Andrén-Sandberg A. Diagnosis and Management of Gallbladder Polyps. Journal of Medical Sciences. 2012;4(5):22-5.

4. Babu B, Dennison A, Garcea G. Management and diagnosis of gallbladder polyps: a systematic review. Langenbecks Arch Surg. 2015;400(4):455-62.

5. Taskin O, Bellolio E, Dursun N, et al. Non-neoplastic polyps of the Gallbladder. A clinicopathologic analysis of 447 cases. Am J. Surg Pathol. 2019;44(4):467-76.

6. Hundal R, Shaffer E. Gallbladder cancer: epidemiology and outcome. Clinical Epidemiology. 2014;6:99-109.

7. Wiles R, Thoeni R, Barbu S, et al. Management and follow-up of gallbladder polyps. Eur Radiol. 2017;27: 3856-66.

8. Elmasry M, Lindop D, Dunne D, et al. The risk of malignancy in ultrasound detected gallbladder polyps: A systematic review. International Journal of Surgery. 2016;9191(16):30270-9.

9. Xu A, Zhang Y, Hu H, et al. Gallbladder Polypoid-Lesions: What Are They and How Should They be Treated? A Single-Center Experience Based on 1446 Cholecystectomy Patients. Journal Gastrointestinal Surg. 2017;21(11):1804-12.

10. Bugosen M, Tagle M, Huerta-Mercado J, et al. Pólipos vesiculares: características clínicas anatomopatológicas en pacientes colescistectomizados en la Clínica Anglo-Americana entre los años 1999-2007. Rev Gastroenterología Perú. 2011;31(1):32-7.

11. Gökay S, Terzio lu C, Murat O, et al. Predictive factors of neoplastic gallbladder polyps: Outcomes of 278 patients. Turk J.Gastroenterol. 2017;28:202-6.

12. Lu D, Radin R, Yung E, et al. Malignant Transformation of a 5-mm Gallbladder Polyp Over 2 Years. A Case Report and Review of Current Literature. Ultrasound Quarterly. 2015;31:66-8.

13. Sun Y, Yang Z, Lan X, et al. Neoplastic polyps in gallbladder: a retrospective study to determine risk factors and treatment strategy for gallbladder polyps. HepatoBiliary Surg Nutr. 2019;8(3):219-27.

14. Rappard V, Bugiani M, Boelens J, et al. Gallbladder and the risk of polyps and carcinoma in metachromatic leukodystrophy. American Academy of Neurology. 2016;87:1-9.

15. Wiles R, Varadpande M, Muly S, et al. Growth rate and malignant potential of small gallbladder polyps- Systematic review of evidence. Journal of the Royal Colleges of Surgeons of Edinburgh and Ireland. 2014;32(4):1-6.

16. Onda S, Futagawa Y, Gocho T, et al. A preoperative scoring system to predict carcinoma in patients with gallbladder polyps. $\mathrm{Di}-$ gestive Surgery. 2019;1-7.

17. Metman M, Olthof P, Van der Wal J, et al. Clinical relevance of gallbladder polyps; is cholecystectomy always necessary? International Hepato-Pancreato-Biliary Association. 2019;22(4):506-10.
18. Tian L, Yang Y, Xiao D, et al. Resection of gallbladder polyps following endoscopic ultrasound-guided cholecystoduodenostomy using a lumen-apposing metal stent. Endoscopy. 2018;50(10):E307-8.

19. Chen Y, Su P, Shen W. Gallbladder polyp treated with endoscopic polypectomy through a cholecystogastrostomy. Endoscopy. 2011;43(2):E88-9.

20. Velidedeo lu M, Çitgez B, Arıkan A, et al. Is it necessary to perform prophylactic cholecystectomy for all symptomatic gallbladder polyps diagnosed with ultrasound? Turk J Surg. 2017;33(1):25-8.

21. Kasle D, Rahnemai-Azar A, Bibi S, et al. Carcinoma in situ in a $7 \mathrm{~mm}$ gallbladder polyp: Time to change current practice? World J Gastrointestinal Endoscopy. 2015;7(9):912-91.

22. Bhatt N, Gillis A, Smoothey C, et al. Evidence based management of polyps of the gallbladder: A systematic review of the risk factors of malignancy. The surgeon, Journal of the Royal Colleges of surgeons of Edinburgh and Ireland. 2016;1(4):278-86.

23. Yin S, Chi J, Liu L, et al. Dual- energy CT to differentiate gallbladder polyps: cholesterol versus adenomatous. Acta Radiológica. 2020;0(0):1-8.

24. Yang X, Liu Y, Guo Y, et al. Utility of radiomics based on contrast-enhanced CT and clinical data in the differentiation of benign and malignant gallbladder polypoid lesions. Abdominal Radiology. 2020;1-10.

25. Choi J-H, Seo D-W, Choi J, et al. Utility of contrast-enhanced harmonic EUS in the diagnosis of malignant gallbladder polyps (with videos). Gastrointestinal Endoscopy. 2013;78(3):484-93.

26. Choi W-B, Lee S-K, Kim M-H, et al. A new strategy to predict the neoplastic polyps of the gallbladder based on a scoring system using EUS. Gastrointestinal Endoscopy. 2000;52(3):372-9.

27. Wennmacker G, Lamberts T, Drenth R, et al. Transabdominal ultrasound or endoscopic ultrasound for diagnosis of gallbladder polyps. Cochrane Database of Systematic Reviews. 2016.

28. Oh T, Park C, Park J, et al. Usefulness of contrast enhanced harmonic- EUS (CEH-EUS) in the diagnosis of neoplastic gallbladder polyps. Gastrointestinal Endoscopy. 2012;75(4S):AB206.

29. Cho J, Park J, Kim Y, et al. Hypoechoic foci on EUS are simple and strong predictive factors for neoplastic gallbladder polyps. Gastrointestinal Endoscopy. 2009;69(7):1244-50.

30. Kim S, Cho J, Kim E, et al. The Efficacy of differential diagnosis between neoplastic and non-neoplastic gallbladder polyps on Color-Flow Doppler Endoscopic Ultrasonograpy. Gastrointestinal Endoscopy. 2017;85(5S):AB336-AB337. 